\title{
Underexpression and abnormal localization of $A T M$ products in ataxia telangiectasia patients bearing ATM missense mutations
}

\author{
Virginie Jacquemin ${ }^{1,2,5}$, Guillaume Rieunier ${ }^{1,2,5}$, Sandrine Jacob ${ }^{1,2,3}$, Dorine Bellanger ${ }^{1,2}$, \\ Catherine Dubois d'Enghien ${ }^{3}$, Anthony Laugé ${ }^{3}$, Dominique Stoppa-Lyonnet ${ }^{1,2,3,4}$ and Marc-Henri Stern ${ }^{\star, 1,2,3}$
}

Ataxia telangiectasia (A-T) is a rare autosomal recessive disorder characterized by progressive cerebellar ataxia, oculocutaneous telangiectasia, immune defects and predisposition to malignancies. A-T is caused by biallelic inactivation of the ATM gene, in most cases by frameshift or nonsense mutations. More rarely, ATM missense mutations with unknown consequences on ATM function are found, making definitive diagnosis more challenging. In this study, a series of 15 missense mutations, including 11 not previously reported, were identified in 16 patients with clinical diagnosis of A-T belonging to 14 families and 1 patient with atypical clinical features. ATM function was evaluated in patient lymphoblastoid cell lines by measuring $H 2 A X$ and $K A P 1$ phosphorylation in response to ionizing radiation, confirming the A-T diagnosis for 16 cases. In accordance with previous studies, we showed that missense mutations associated with A-T often lead to ATM protein underexpression (15 out of 16 cases). In addition, we demonstrated that most missense mutations lead to an abnormal cytoplasmic localization of ATM, correlated with its decreased expression. This new finding highlights ATM mislocalization as a new mechanism of $A T M$ dysfunction, which may lead to therapeutic strategies for missense mutation associated A-T.

European Journal of Human Genetics (2012) 20, 305-312; doi:10.1038/ejhg.2011.196; published online 9 November 2011

Keywords: ATM; missense mutation; ataxia telangiectasia; pathogenic mutation; subcellular localization

\section{INTRODUCTION}

Ataxia telangiectasia (A-T) (MIM\#208900) is a rare genetic disorder with autosomal recessive inheritance. ${ }^{1} \mathrm{~A}-\mathrm{T}$ features include cerebellar ataxia, oculomotor apraxia, oculocutaneous telangiectasia, immune deficiency, elevated serum $\alpha$-fetoprotein level and acquired 7 and 14 chromosome translocations in the lymphocyte karyotype. In addition, A-T is characterized by clinical and cellular hypersensitivity to ionizing radiation (IR) and an increased risk of cancer. ${ }^{2,3}$ A-T may be difficult to diagnose, particularly in young patients before the appearance of all features of the disease and in milder or atypical cases. Identification of the molecular defect is critical in such cases.

A-T disorder is due to biallelic inactivation of the ATM gene (MIM\#607585). ${ }^{4}$ The ATM gene product is a 3056 amino-acid protein that has a central role in the cellular response to DNA damage. This serine/threonine protein kinase belongs to the phosphoinositide 3-kinase-related protein kinase family. ATM is mainly activated when DNA double-strand breaks occur, and regulates a complex signaling cascade involved in the detection and repair of this type of DNA damage (for review Lavin ${ }^{5}$ ). ATM is predominantly located in the nucleus, although a fraction of ATM has been found associated with peroxisomes. ${ }^{6}$ Interestingly, functions independent of DNA damage signaling have been recently reported. ${ }^{7-9}$

A wide variety of mutations have been identified scattered over the entire coding sequence of the ATM gene. The majority of these mutations are frameshift or nonsense mutations, ${ }^{10-13}$ accounting in our diagnosis series for $73 \%$ of cases, ${ }^{14}$ suggesting that the classical A-T phenotype is due to total loss of ATM protein. ATM missense mutations could be responsible for typical, ${ }^{13}$ or milder phenotypes, ${ }^{15,16}$ but the consequences of many missense mutations not previously associated with A-T remain unknown. Bioinformatics tools are helpful in the latter case but generally insufficient to definitely establish the A-T diagnosis, and direct biological analyses are necessary to determine the pathogenic character of such mutations. Recent studies showed that functional consequences of ATM missense variants can be assessed by modeling ATM mutations and testing their deleterious effects in complementation assays. ${ }^{17-19}$ In both studies, most ATM missense mutations in A-T were associated with expression defects and/or kinase activity deficiencies.

In this study, we report the analysis of a series of missense mutations of the ATM gene, including 11 not previously reported, identified in 16 patients with clinical diagnosis of A-T belonging to 14 families and 1 patient with atypical clinical features. Deleterious consequences of these mutations were found in all but one case by functional assessments of ATM activity, strengthening the A-T diagnosis for 16 patients. We confirmed that ATM missense mutations are associated with protein underexpression in all but one A-T patients. Furthermore, we showed for the first time that this underexpression was associated with an abnormal cytoplasmic localization in 12 of the

\footnotetext{
${ }^{1}$ Institut Curie, Centre de Recherche, Paris, France; ${ }^{2}$ INSERM U830, Paris, France; ${ }^{3}$ Institut Curie, Hôpital, Service de Génétique, Paris, France; ${ }^{4}$ Faculté de médecine Université Paris-Descartes, Paris, France

*Correspondence: Dr MH Stern, INSERM U830, Institut Curie, Centre de Recherche, 26 rue d’UIm, F-75248 Paris cedex 05 , France. Tel: +33 156246646 ; Fax: +33 156246630 ; E-mail: marc-henri.stern@curie.fr

5 These authors contributed equally to this work.

Received 26 January 2011; revised 16 September 2011; accepted 22 September 2011; published online 9 November 2011
} 
$16 \mathrm{~A}-\mathrm{T}$ patients bearing missense mutations. These data suggest a new mechanism accounting for ATM deficiency, which may lead to new therapeutic strategies.

\section{MATERIALS AND METHODS}

\section{Patient selection and mutation detection}

Patients with suspected A-T were referred to the Institut Curie genetic laboratory for ATM mutation screening. Blood samples were used for genetic analysis and for lymphoblastoid cell line (LCL) establishment with the informed consent of patients or their parents for young patients. According to Micol et al, ${ }^{14}$ clinical diagnosis of A-T was made when patients presented ataxia and/or oculomotor apraxia and at least two of the following manifestations: oculocutaneous telangiectasia, recurrent infections, low serum IgA level, high serum $\alpha$-fetoprotein level (AFP) or karyotype abnormalities such as translocations or inversions of chromosomes 7 and 14. This study include 17 patients harboring ATM missense mutations: 16 with clinical diagnosis of A-T and 1 case (38437A1) with non-defined diagnosis, but presenting some features of A-T (ataxia and increased level of AFP).

The 62 coding exons of ATM and an average of 30 nucleotides spanning each exon/intron junction were analyzed by direct sequencing of genomic DNA (RefSeq U82828.1). Primer sequences and PCR amplification conditions are available upon request. For compound heterozygote mutations, the presence of one mutation on each parental allele (in trans) was verified when parental DNA was available. The search for large gene rearrangements was performed by semiquantitative PCR, using the Multiplex Ligation-dependent Probe Amplification (MLPA) kit (SALSA MLPA KIT P041/P042 ATM, MRC-Holland, Amsterdam, The Netherlands). As nucleotide variations can lead to splicing abnormalities, the genomic sequence environment of each DNA variant was analyzed using Splice Site Prediction by Neural Network (NNSPLICE available at http://www.fruitfly.org/seq_tools/splice.html), MaxEntScan (MES available at http://genes.mit.edu/burgelab/maxent/Xmaxentscan_scoreseq. html). ${ }^{20}$ Direct sequencing of the cDNA region surrounding the mutation was performed when a splicing defect was predicted. The ATM mutation detection rate in the A-T patients has been estimated to be $96 \%$ (292 mutations found in a series of 305 alleles tested). ${ }^{14}$

Patients were selected for this study when at least one ATM missense mutation was identified together with available LCL and a clinical diagnosis compatible with A-T.

\section{Cell lines}

Epstein-Barr virus-immortalized LCLs were established by Genethon (http:// www.genethon.fr/). They were grown in RPMI 1640 (Invitrogen, Oxon, UK) supplemented with $10 \%$ fetal calf serum (FCS; Invitrogen), penicillin $(0.1 \mu \mathrm{g} / \mathrm{ml})$ and streptomycin $(0.1 \mu \mathrm{g} / \mathrm{ml}$; Invitrogen $)$ at $37^{\circ} \mathrm{C}$ in a humidified incubator with $5 \% \mathrm{CO}_{2}$.

One A-T LCL carrying biallelic ATM inactivating mutations (A-T; c.2483delA/p.Lys828SerfsX8 and c.3511C > T/p.Gln1171X) and nine LCLs derived from normal individuals (http://www.genethon.fr/) were used as A-T and wild-type (WT) controls, respectively.

\section{Response to ionizing radiation}

Cell lines in exponential growth were seeded at a density of $150000 \mathrm{cells} / \mathrm{ml}$ in $10 \mathrm{ml}$ RPMI 10\% FCS in polypropylene round-bottom tubes and irradiated with a dose of 5 Gy using a Cesium $\gamma$-irradiator IBL137 (1.73 Gy/min for $175 \mathrm{~s})$ and incubated at $37^{\circ} \mathrm{C}$. Cells were either analyzed for P-KAP1 (see next paragraph) or fixed at 0 and $1.5 \mathrm{~h}$ after irradiation in $70 \%$ ethanol for FACS analysis of $H 2 A X$ phosphorylation, according to Olive et al with minor modifications. ${ }^{21}$ Briefly, cells were resuspended in Tris-buffered saline (TBS), 0.5\% Triton, 4\% FCS for $10 \mathrm{~min}$ at $4{ }^{\circ} \mathrm{C}$ and incubated with anti-phosphoSer139-H2AX antibody (05-636, $1 / 1000$, Millipore, Billerica, MA, USA) for $1 \mathrm{~h}$ at $37^{\circ} \mathrm{C}$. After washing in TBS, cells were incubated with FITC-coupled mouse secondary antibody for $1 \mathrm{~h}$ at room temperature (RT). Labeled cells were characterized on a FACS CantoTMII (BD Biosciences, Franklin Lakes, NJ, USA) and the results were analyzed with Flowjo software (Tree Star, Ashland, OR, USA; v. 8.8.2). Significance of each sample versus the WT control was tested by Student's unpaired $t$-test.

\section{Western blot analysis}

Cells were collected by centrifugation at 1500 r.p.m. for $10 \mathrm{~min}$ at $4{ }^{\circ} \mathrm{C}$ and washed in cold phosphate-buffered saline (PBS). Cell pellets were lysed in radioimmunoprecipitation assay buffer $(100 \mathrm{~mm}$ Tris- $\mathrm{HCl} / \mathrm{pH} 7.5,0.1 \mathrm{M} \mathrm{NaCl}$, $1 \mathrm{~mm}$ EDTA, $1 \%$ Triton, $0.5 \%$ sodium deoxycholate $0.1 \%$ sodium dodecyl sulfate (SDS)), supplemented with $\mathrm{NaF}, \mathrm{Na}_{3} \mathrm{VO}_{4}$, phenylmethanesulfonylfluoride (PMSF) and a cocktail of protease inhibitors (Complete, Roche, Basel, Switzerland). A total of $80 \mu \mathrm{g}$ of protein extracts were separated in a 5\% SDSpolyacrylamide gel (SDS-PAGE) and transferred to nitrocellulose membranes. Membranes were blocked in 5\% non-fat dry milk for $1 \mathrm{~h}$ at RT and incubated overnight at $4{ }^{\circ} \mathrm{C}$ with the primary antibodies at the following concentrations: ATM (A300-136, 1/2500, Bethyl Laboratories, Montgomery, TX, USA), phosphoSer824-KAP1 (A300-767A, 1/1000, Bethyl), $\beta$-actin (A-5316, 1/20000, Sigma) and $\alpha$-adaptin (610502, 1/1000, BD Biosciences) used as a control for protein loading. The secondary antibody coupled to horseradish peroxidase (anti-goat: sc-2020, Santa Cruz Biotechnology, Santa Cruz, CA, USA; anti-mouse: NA931V, and anti-rabbit: NA934V, both from GE Healthcare, Velizy, France) was revealed using the ECL kit (34080, Pierce, Rockford, IL, USA) and band intensities were quantified using ImageJ software (imagej.nih.gov/ij/).

For ATM subcellular localization, nuclear and cytoplasmic extracts were prepared using the NE-PER reagents (Pierce) according to the manufacturer's instructions. Corresponding fractions of the cytoplasmic and nuclear extracts from equal cell numbers were separated in 5\% SDS-PAGE gel. The purity of each extract was monitored by MEK1/2 (\#9122, 1/1000, Cell Signaling Technology, Danvers, MA, USA) and INI1 (BAF47-25, 1/1000, BD Biosciences) as specific markers of the cytoplasmic and nuclear compartments, respectively.

\section{Immunofluorescence}

Cells were cytospined and fixed for $20 \mathrm{~min}$ in PBS 4\% paraformaldhyde (PFA), washed in PBS, permeabilized for $10 \mathrm{~min}$ in PBS $0.1 \%$ SDS. Cells were washed in PBS, blocked for $1 \mathrm{~h}$ in PBS $10 \%$ bovine serum albumin (BSA), washed in PBS and incubated with anti-ATM antibody (NB 100-104, 1/250, Novus Biologicals, Littleton, CO, USA) for $1 \mathrm{~h}$ at RT. Cells were washed in PBS, incubated with secondary antibody Alexa Fluor 488 (A21206, 1/50, Invitrogen) for $1 \mathrm{~h}$ at RT in the dark. Slides were air-dried and mounted in VectaShield (H1200, Vector Laboratories, Burlingame, CA, USA) with DAPI.

Images were captured using a MicroMax camera (Princeton Instrument, Trenton, NJ, USA) connected to a Zeiss Axioplan 2 fluorescence microscope (Carl Zeiss, Le Pecq, France) with a $100 \times / 1.3$ oil immersion objective. Acquisitions were made using the MetaMorph software (Universal Imaging, Burbank, CA, USA). The images were then imported in ImageJ, pseudocolored and merged using the same software.

\section{Quantitative RT-PCR analysis}

RNA was extracted using Trizol Reagent (Invitrogen). ATM expression with respect to the housekeeping gene $G A P D H$ was evaluated by real-time quantitative PCR using the SYBR system according to the manufacturer's protocol (Applied Biosystems, Carlsbad, CA, USA). Amplification of $50 \mathrm{ng}$ of DNasedigested retrotranscripted RNA was performed using the following primers: ATM_F 5'-CCTTGGCTACAGATTGCAACC-3', ATM_R 5'-ACTTCCGTAAGGCATCGTAACAC-3', GAPDH_F 5'-TGCACCACCAACTGCTTAGC-3' GAPDH_R 5'-GGCATGGACTGTGGTCATGAG-3'. Fluorescence intensity was measured by a 7500 Real-Time PCR system (Applied Biosystems) and normalized by the $2^{-\Delta \Delta C T}$ method. Gene expression in three control cell lines was used for baseline expression. Significance of each sample versus the WT controls was tested by Student's unpaired $t$-test.

\section{RESULTS}

\section{Patients and mutations}

A series of 17 patients with clinical features compatible with A-T, belonging to 15 families ( 2 patients in 2 families) and presenting at least 1 ATM missense mutation with unknown consequences on ATM function was studied. The clinical and laboratory features of these patients are reported in Table 1 . All patients presented ataxia with variable ages at onset from 1 to 10 years old and half presented at least 5 of the $6 \mathrm{~A}-\mathrm{T}$ criteria. One patient (38437A1) presented only two 
Table 1 Clinical and laboratory features of the 17 patients bearing ATM missense mutations

\begin{tabular}{|c|c|c|c|c|c|c|c|}
\hline Patient & $\begin{array}{l}\text { Cerebellar syndrome } \\
\text { (age at onset) }\end{array}$ & $\begin{array}{l}\text { Telangiectasia } \\
\text { (age at onset) }\end{array}$ & $\begin{array}{c}\text { 7-14 Rearrangements } \\
\text { (7-14/number } \\
\text { of mitoses) }\end{array}$ & $\lg A^{\mathrm{a}}$ & $\begin{array}{l}\text { Recurrent } \\
\text { infections }\end{array}$ & $\begin{array}{c}\text { AFP }(\mathrm{ng} / \mathrm{ml}) \\
\quad(\text { normal } \\
<10 \text { after } 2 \mathrm{y})\end{array}$ & Other symptoms \\
\hline $32760 A 2$ & Ataxia (2y) & + & $+(5 / 67)$ & $5 \%$ & + & 275 at $9 y$ & \\
\hline $38633 A 1$ & Ataxia (6y) oculomotor apraxia (9y) & + & $+(2 / 50)$ & $10 \%$ & No & $<10$ & \\
\hline 9793A1 & Ataxia, oculomotor apraxia (8y) & + & $+(6 / 30)$ & $8 \%$ & No & 322 at $43 y$ & $\begin{array}{l}\text { Hypersensibility to radia- } \\
\text { tion, hand dysgenesis, } \\
\text { myoclonies, breast cancer } \\
\text { at } 43 y\end{array}$ \\
\hline 38892A2 & Ataxia (1 y) & + & $+(5 / 50)$ & $5 \%$ & + & 140 at $5 y$ & \\
\hline 38892A6 & Ataxia (1 y) & + & No & $24 \%$ & No & 322 at $8 y$ & \\
\hline $32753 A 6$ & Ataxia (1 y) & + & $+(5 / 50)$ & No decrease & No & 56 at $9 y$ & \\
\hline 38437A1 & Ataxia (3y) & Absent at $7 y$ & No & No decrease & No & 39 at $3 y$ & $\begin{array}{l}\text { Cerebral palsy facial } \\
\text { dysmorphy }\end{array}$ \\
\hline $38563 A 1$ & Ataxia (2y) & + & $+(2 / 25)$ & No decrease & NA & 43 at $3 y$ & \\
\hline 39039A4 & Ataxia (2y) oculomotor apraxia & Absent at $5 y$ & $+(2 / 31)$ & $2.5 \%$ & + & 37 at $6 y$ & \\
\hline 39137A1 & Ataxia (1 y) oculomotor apraxia & + & $+(5 / 100)$ & No decrease & No & 40 at $10 y$ & \\
\hline $40362 \mathrm{~A} 1^{\mathrm{b}}$ & Ataxia $(10 y)$ & Absent at $31 \mathrm{y}$ & $+(5 / 100)$ & ND & No & 92 at $29 y$ & \\
\hline $41832 \mathrm{~A} 1$ & Ataxia (1 y) & + & $+(2 / 80)$ & $25 \%$ & + & 687 at $7 y$ & \\
\hline $53011 A 1$ & Ataxia (1 y) oculomotor apraxia & $+(5 y)$ & $+(1 / 30)$ & No decrease & No & 42 at $5 y$ & \\
\hline $56525 A 2$ & Ataxia (1 y) no oculomotor apraxia (2y) & + & No $(50)$ & $15 \%$ & + & 80 at $2 y$ & \\
\hline $60669 A 1$ & Ataxia (1 y) & $+(6 y)$ & $+(5 / 100)$ & $\begin{array}{c}\text { No decrease at } \\
6 y\end{array}$ & No & 26 at $6 y$ & \\
\hline $63021 \mathrm{~A} 1$ & Ataxia (2y) & + & $+(2 / 100)$ & $2 \%$ & NA & 337 at $15 y$ & Amyotrophy \\
\hline $63021 \mathrm{~A} 2$ & Ataxia (1 y) & + & ND & $7 \%$ & + & 111 at $10 y$ & \\
\hline
\end{tabular}

Abbreviations: AFP, $\alpha$-fetoprotein; NA, not available; ND, not done; WT, wild type; y, year old.

aExpressed as a percentage of age-matched WT controls.

bSister with similar mild A-T.

criteria and additional clinical features such as facial dysmorphia and cerebral palsy, which are not typical in A-T. Karyotypes were available for at least one patient per family in the 15 families and were characteristic of A-T in 13 families (Table 1).

As shown in Table 2, 15 different ATM missense mutations were found in these patients, including 11 mutations never previously described in A-T patients (http://chromium.liacs.nl/LOVD2/home.php, http:// www.hgmd.cf.ac.uk/ac/index.php). Half of these mutations (7 out of 15 mutations) were located in codons 2401 to 3056, containing the phosphoinositide 3-kinase catalytic domain; a single mutation (p.Arg386Gly in case 53011A1) was within the Nuclear Localization Sequence (NLS, ${ }^{385} \mathrm{KRKK}^{388}$ ). ${ }^{22}$ Using the A-GVGD algorithm (http:// agvgd.iarc.fr/agvgd_input.php), ${ }^{23}$ which predicts substitution effects based on assessment of chemical dissimilarity between residues and phylogenetic conservation, 3 missense mutations were considered to be unlikely to be pathogenic (score $\leq \mathrm{C} 15$ ), 2 moderately likely to be pathogenic (C25 or C35) and 10 likely to be pathogenic (score $\geq \mathrm{C} 45$ ). No consequence of these missense mutations on splice was evidenced. In addition, two single nucleotide polymorphisms (SNP) leading to an amino-acid change were associated with missense mutations in patient 41832A1 (c.378T >A;p.Asp126Glu as rs2234997) and in patient 38437A1 (c.3383A > G; p.Gln1128Arg as rs56398245).

Direct evaluation of the effects of missense mutations was possible in 14 of the 15 families, as these mutations were either homozygous or associated with a clearly pathogenic allele (nonsense, frameshift or splicing mutations). Separate analysis of each missense mutation was not possible in one cell line carrying two different missense mutations in trans.

\section{ATM function}

To confirm the A-T diagnosis in these families, functional approaches assessing the capability of $A T M$ to phosphorylate $H 2 A X$ and KAP1 were performed. FACS analysis of $H 2 A X$ phosphorylation $(\gamma H 2 A X)$ after IR was shown to correctly discriminate A-T cell lines from cell lines derived from normal individuals as well as from related syndromes such as Nijmegen Breakage Syndrome, A-T-like disease, Friedreich ataxia and ataxia with oculomotor apraxia type 2 (AOA2). ${ }^{24}$ LCLs were irradiated at a dose of 5 Gy and the H2AX phosphorylation status was measured by FACS at the peak of phosphorylation $(1.5 \mathrm{~h})$. As shown in Figures $1 \mathrm{a}$ and $\mathrm{b}$, the intensity of the $\gamma H 2 A X$ peak was markedly decreased in an A-T control cell line as compared with nine WT cell lines. The $16 \mathrm{~A}-\mathrm{T}$ cell lines with ATM missense mutations presented a significantly decreased response to IR similar to that of an A-T control cell line. Interestingly, the cell line derived from case 38437Al with undefined diagnosis presented normal response to IR.

We next assessed the phosphorylation of another ATM-dependent target, KAP1, in response to IR. ${ }^{25}$ Consistently with the $\gamma H 2 A X$ assay, we found an impaired response to IR for all ATM mutant cell lines but 38437A1 (see Figure 1c and Supplementary Figure 1). Interestingly, two cell lines (40362A1 and 53011A1) presented residual phosphorylation of KAP1. Again, the cell line derived from case 38437A1 with undefined diagnosis presented normal response to IR on KAP1 phosphorylation.

\section{ATM expression}

To test the effect of each missense mutation on ATM expression, western blot analysis was performed on LCLs established from patients (Figure 2). The antibody used for ATM detection specifically recognizes residues 2550-2600, an epitope not directly modified by the mutations in this series of patients. As expected, no ATM protein was detected in the control A-T cell line. Level of ATM protein expression was markedly decreased in 3 cases $(<20 \%$ expression of the ATM level 
Table 2 ATM status in 17 LCLs bearing ATM missense mutations

\begin{tabular}{|c|c|c|c|c|c|c|}
\hline & Genomic & Protein & $\begin{array}{l}\text { Consequences and } \\
\text { A-GVGD score }\end{array}$ & $\begin{array}{c}\text { ATM transcript } \\
\% \text { of } W T^{\mathrm{a}}\end{array}$ & $\begin{array}{c}\text { ATM protein } \\
\% \text { of } W T^{\mathrm{a}}\end{array}$ & $\begin{array}{l}\text { Nuclear ATM } \\
\% \text { of total }\end{array}$ \\
\hline $32760 A 2$ & c. $7462 \mathrm{~T}>\mathrm{C}$ & p.Cys2488Argb & $\mathrm{C} 65$ & $54 \pm 5$ & $17 \pm 14$ & $20 \pm 18$ \\
\hline \multirow[t]{2}{*}{$38633 A 1$} & c. $7570 \mathrm{G}>\mathrm{C}$ & p.Ala2524Pro ${ }^{\mathrm{b}}$ & $\mathrm{C} 25$ & $55 \pm 5$ & $45 \pm 12$ & $58 \pm 6$ \\
\hline & c. $3663 \mathrm{G}>\mathrm{A}$ & p.Trp1221X & Stop & & & \\
\hline 9793A1 & c. $5189 \mathrm{G}>\mathrm{T}$ & p.Arg1730Leu & $\mathrm{CO}$ & $51 \pm 4$ & $11 \pm 5$ & $11 \pm 6$ \\
\hline $38892 A 6$ & c.7879T > C (Hmz) & p.Tyr2627His & $\mathrm{C} 65$ & $78 \pm 12$ & $54 \pm 30$ & $40 \pm 18$ \\
\hline \multirow[t]{2}{*}{ 32753A6 } & c. $8124 \mathrm{~T}>\mathrm{A}$ & p.Asp2708Glu & $\mathrm{C} 35$ & $55 \pm 8$ & $8 \pm 4$ & $40 \pm 9$ \\
\hline & c.8251_8254del4 & p.Thr2751_Ile2752 > SerfsX54 & Frameshift & & & \\
\hline \multirow[t]{3}{*}{$38437 A 1$} & $c .488 \mathrm{~A}>\mathrm{G}$ & p.Gln163Arg & $\mathrm{CO}$ & $85 \pm 17$ & $87 \pm 17$ & $58 \pm 10$ \\
\hline & c. $3383 \mathrm{~A}>\mathrm{G}$ & p.GIn1128Arg & $\mathrm{co}$ & & & \\
\hline & c.8419-54del6 & NA & Unknown & & & \\
\hline $39137 A 1$ & c. $3576 \mathrm{G}>\mathrm{A}$ & p.Ser1135_Lys1192del & Exon skipping & & & \\
\hline $40362 A 1$ & c. $6203 \mathrm{~T}>\mathrm{C}(\mathrm{Hmz})$ & p.Leu2068Ser & C55 & $108 \pm 21$ & $59 \pm 20$ & $48 \pm 10$ \\
\hline \multirow[t]{2}{*}{ 41832A1 } & c. $378 \mathrm{~T}>\mathrm{A}^{\mathrm{d}}(\mathrm{Hmz})$ & p.Asp126Glu & $\mathrm{co}$ & $83 \pm 30$ & $29 \pm 14$ & $13 \pm 18$ \\
\hline & c. $8737 \mathrm{G}>\mathrm{T}(\mathrm{Hmz})$ & p.Asp2913Tyr & $\mathrm{C} 65$ & & & \\
\hline \multirow[t]{2}{*}{$53011 \mathrm{~A} 1$} & c. $1156 A>G$ & p.Arg386Gly & $\mathrm{C} 45$ & $100 \pm 0$ & $67 \pm 14$ & $38 \pm 11$ \\
\hline & c.8461_8463delATG & p.Met2821del & Amino acid deletion & & & \\
\hline $56525 A 2$ & c. $7985 \mathrm{~T}>\mathrm{A}(\mathrm{Hmz})$ & p.Val2662Asp & $\mathrm{C} 65$ & $96 \pm 25$ & $44 \pm 12$ & $17 \pm 22$ \\
\hline \multirow[t]{2}{*}{ 60669A1 } & c. $6491 \mathrm{~A}>\mathrm{C}$ & p.Glu2164Ala & $\mathrm{C} 65$ & $82 \pm 12$ & $109 \pm 22$ & $59 \pm 5$ \\
\hline & c. $6115 \mathrm{G}>\mathrm{A}$ & p.Glu2039Lys ${ }^{b}$ & $\mathrm{C} 55$ & & & \\
\hline $63021 \mathrm{~A} 1$ & c.7985T>A (Hmz) & p.Val2662Asp & $\mathrm{C} 65$ & $80 \pm 1$ & $27 \pm 16$ & $23 \pm 10$ \\
\hline $63021 A 2$ & c.7985T>A (Hmz) & p.Val2662Asp & $\mathrm{C} 65$ & $107 \pm 21$ & $32 \pm 15$ & $23 \pm 18$ \\
\hline WTs & WT & WT & NA & $100 \pm 21$ & $100 \pm 9$ & $60 \pm 13$ \\
\hline
\end{tabular}

Abbreviations: A-T: ataxia telangiectasia; Hmz: homozygous mutation; LCL, lymphoblastoid cell line; NA: not applicable; ND: not done; WT, wild type.

Gray shading represents significant differences with WT.

a Expressed as the mean \pm SD of three independent experiments.

bAmino-acid changes previously reported in A-T patients.

crs56398245.

drs2234997.

found in WT cell lines), moderately decrease in 12 cases (20 to $61 \%$ of normal ATM level) and normal in 2 cases (38437A1 and 60669A1; see Table 2). As expected, the LCL derived from the non-A-T patient 38437A1 presented normal ATM expression. Cell lines from different individuals or families carrying the same missense mutation presented similar levels of ATM expression. In comparison, MRE11, aprataxin and senataxin analyzed for differential diagnosis by western blotting were found normal (data not shown).

These expression defects could be due to transcriptional deregulation, which was tested by quantitative RT-PCR in mutant cell lines (Table 2). ATM transcripts were markedly decreased (29\% of WT level) in the A-T control cell line carrying nonsense mutations, as a possible consequence of nonsense-mediated mRNA decay. ${ }^{26}$ Accordingly, patient cell lines carrying an allele with nonsense mutations or splice defects introducing a frameshift had a twofold decrease in ATM transcripts (range $46-53 \%$ of WT level). Cell lines carrying only missense mutations or a short in-frame deletion had ATM transcripts in a range from 60 to $108 \%$ of WT level. These results demonstrate that transcriptional deregulation does not explain the decrease in ATM protein observed in LCLs bearing missense mutations.

\section{ATM localization}

ATM defects could also be related to its abnormal location..$^{22}$ Normal nuclear fraction was found to represent more than half of total ATM $(60 \pm 13 \%)$ in four WT cell lines (Figure 3a and Supplementary Figure 2). This fraction was measured in the series of patient cell lines and found significantly decreased (nuclear fraction representing $11-48 \%$ of total $A T M$ ) in 12 out of the 17 cell lines, all presenting a low expression of ATM. Consistently with western blot analysis, an abnormal cytoplasmic localization of ATM was evidenced by immunofluorescence for the 56525A2, 63021A2, 38563A1 and 53011A1 cell lines, which expressed sufficient ATM protein for detection (Figure 3b). No ATM could be detected in the A-T control cell line, demonstrating the specificity of the immunofluorescence (Figure $3 \mathrm{~b}$ ). Five of the cell lines presented no significantly abnormal localization of ATM (nuclear fraction: 39-59\%), including the two cell lines with no decrease in ATM expression. These data suggested a link between ATM underexpression and abnormal cytoplasmic localization. Interestingly, the total ATM protein level was found to positively correlate with its nuclear localization (Pearson's test, $r=0.716, P<0.0006$; Figure $3 \mathrm{c}$ ). This correlation was still significant in the subgroup with 

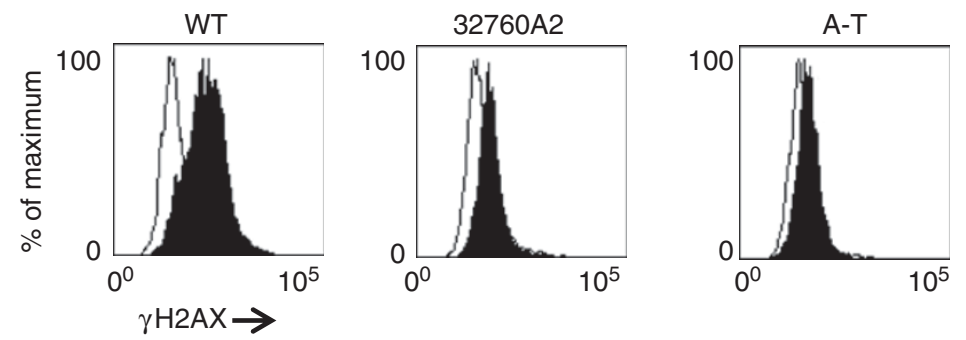

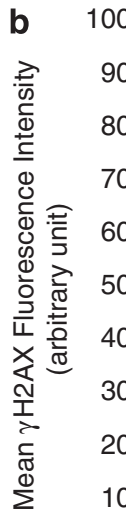
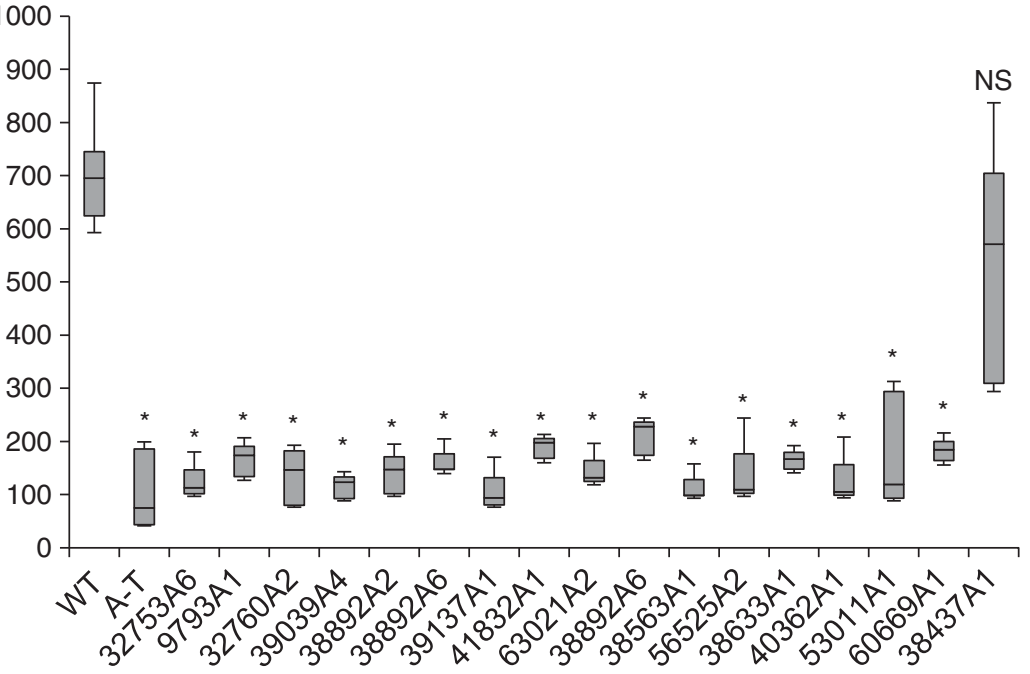

C

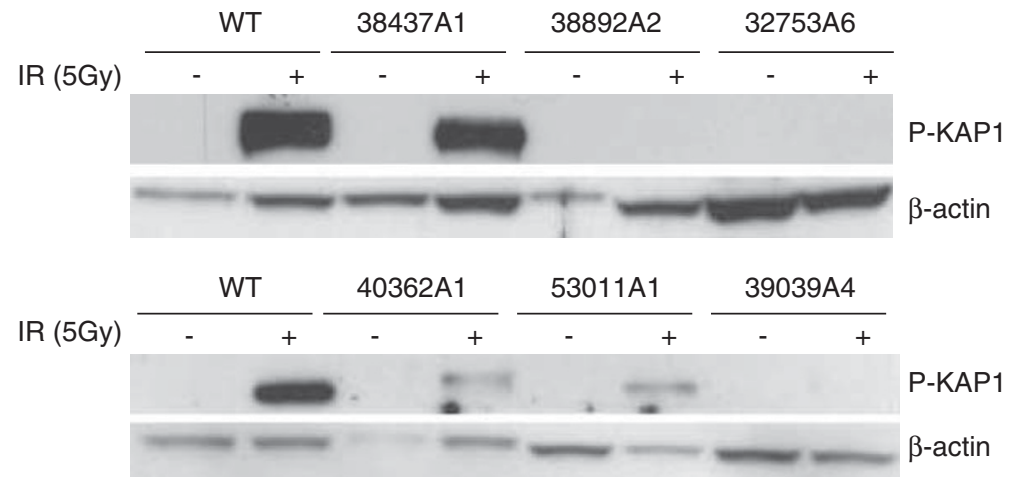

Figure 1 Response to IR. (a) $H 2 A X$ phosphorylation in response to IR, $1.5 \mathrm{~h}$ after $5 \mathrm{~Gy}$ irradiation. $H 2 A X$ fluorescence intensity was analyzed as an FL1-FITC histogram. Filled histogram: irradiated cells; empty histogram: untreated cells. (b) H2AX phosphorylation was measured by FACS and expressed as the difference with basal level (OGy). The box plot represents the mean and SD of at least three experiments. WT represents the mean and SD of nine normal cell lines. The significance of each sample versus WT is indicated above each column; NS, not significant; ${ }^{*} P<0.001$. (c) Analysis of $K A P 1$ phosphorylation $1 \mathrm{~h}$ after $5 \mathrm{~Gy}$ irradiation in cell lines derived from 17 patients bearing ATM missense mutations. $\beta$-actin is used as a loading control. WT cell line; A-T: A-T cell line carrying two truncating mutations in the ATM gene.

decreased ATM values (Pearson's test, $r=0.614, P<0.02$ ). This suggests that mutated $A T M$ decreased protein level and mislocalization may be linked to a common mechanism. A noteworthy exception of this rule was the 386633Al cell line, presenting decreased ATM expression and no abnormal protein localization.

\section{DISCUSSION}

Determining the clinical significance of missense variants is a recurrent problem in medical genetics. In this study, we characterized 17 LCLs bearing ATM missense mutations for ATM function, expression and localization. A total of 16 of these cases had a clinical diagnosis of A-T according to Micol et al, ${ }^{14}$ as they presented (1) ataxia and at least two of the other five signs listed above: (2) oculocutaneous telangiectasia,
(3) recurrent infections, (4) low serum IgA level, (5) high serum AFP and (6) specific karyotype abnormalities. For these 16 A-T cases, ATM function was consistently impaired and for 15 out of 16 cases either ATM expression level or cellular location or both were abnormal, strengthening the clinical diagnosis of $\mathrm{A}-\mathrm{T}$. The additional patient 38437Al was included in this study because of some clinical features of A-T and the detection of two ATM missense mutations and a $6 \mathrm{bp}$ intronic deletion without predicted splicing effects. However, these missense mutations had rather conservative amino-acid change (C0 A-GVGD score) and one was recently described as a SNP with a minor allelic frequency of 0.023 (rs56398245, http://www.ncbi.nlm. nih.gov/SNP/). Her clinical features were ataxia and elevated AFP (eightfold), but no telangiectasia at the age of 7 years old, no 


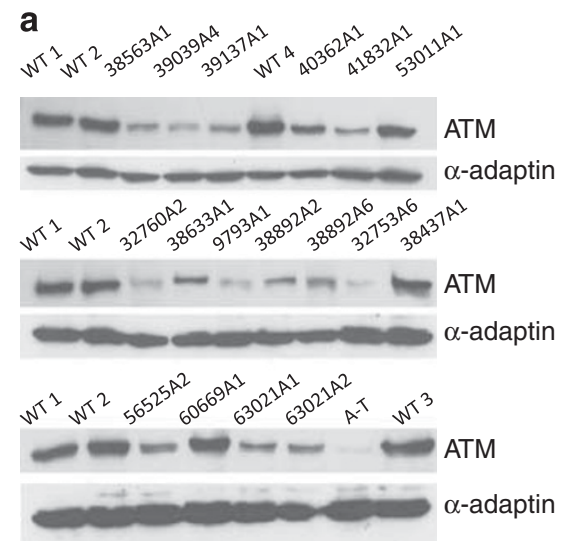

b

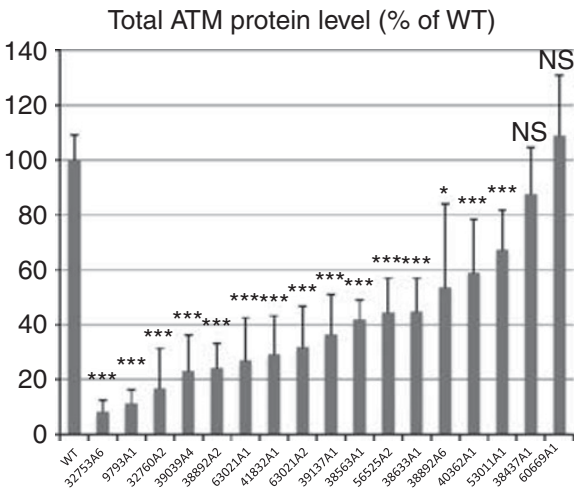

Figure 2 Western blot analysis of ATM. (a) Analysis of ATM protein level in cell lines derived from 17 patients bearing ATM missense mutations. $\alpha$-Adaptin is used as a loading control. WT1-3 are WT cell lines, WT4 carries ATM SNP rs1800058 at the heterozygote state and an A-T cell line carries two truncating mutations in the ATM gene. This experiment is representative of at least three assays per sample. (b) Quantification was performed using ImageJ software. The histogram and error bars represent the mean and SD of ATM levels related to $\alpha$-adaptin calculated from three independent western blots. The significance of each sample versus the control is indicated above each column; NS, not significant; ${ }^{*} P<0.05$ and ${ }^{* *} P<0.001$.

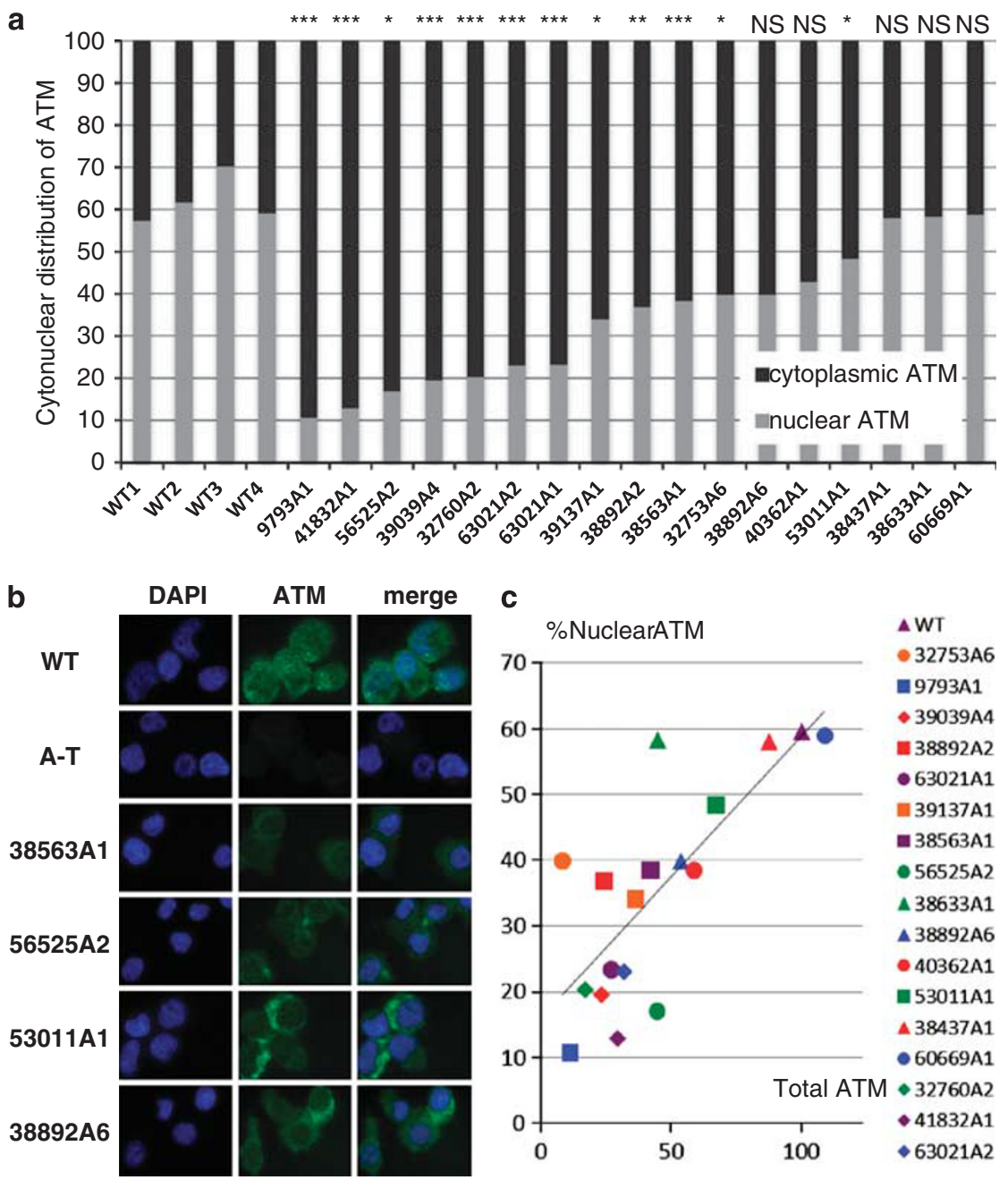

Figure 3 Analysis of ATM subcellular localization. (a) The histograms represent the mean fraction of nuclear (light gray) versus cytoplasmic (dark gray) ATM calculated from three independent experiments. WT1-3 are WT cell lines, WT4 carries ATM SNP rs1800058 at the heterozygote state. Samples showing a significantly lower nuclear fraction as compared with WT controls are indicated by; NS, not significant; ${ }^{*} P<0.05,{ }^{* *} P<0.01$ and ${ }^{* * *} P<0.001$. (b) Localization of ATM by immunofluorescence. Representative images of four ATM missense mutation bearing cell lines are shown, together with WT and A-T cell lines as positive and negative controls. DNA is stained with DAPI (magnification $\times 1000$ ). (c) Graph illustrating the correlation between ATM protein expression (expressed as percentage of normal ATM level on the $x$-axis) and nuclear fraction (expressed as percentage of total ATM level on the $y$-axis). 
karyotype abnormality and no immune deficiency. Furthermore this patient presented facial dysmorphia and cerebral palsy that are rarely associated with A-T. The cell line derived from this patient displayed neither biochemical nor functional ATM defects, suggesting that these ATM missense mutations are not causative. Differential diagnosis includes AOA2, which is also associated with mild elevation of AFP, but no abnormality of SETX was detected and diagnosis is still pending.

In this study, biochemical and functional analyses supported the A-T diagnosis that was made on the basis of clinical features for 16 patients all presenting abnormal ATM expression, localization or ATM dysfunction and to exclude the A-T diagnosis for the patient with a pending diagnosis as she presented no ATM biochemical or functional abnormalities. Our series included 12 patients with missense mutations and without any detectable ATM kinase activity and accordingly, typical severe clinical features. The two remaining patients had milder or atypical A-T phenotypes. Case 40362A1 had a late onset ataxia (10 years) with neither telangiectasia nor clinical immunodeficiency, associated with a slight decrease in ATM expression, no $A T M$ mislocalization and more importantly a residual $\mathrm{P}-K A P 1$ response to IR, which may explain the milder disease. Case 9793A1 was a female patient with late onset ataxia, who survived until the age of 44 when she succumbed from acute adverse effects following breast cancer radiotherapy. Contrasting with the mild phenotype, this case was associated with a marked decrease in ATM protein $(11 \%$ of the WT), a severe abnormal localization of ATM (with a nuclear fraction of $11 \%$ ) and a response to IR, which was similar to an A-T control cell line. Two genetic mutations were found: one leading to an in-frame deletion of 29 amino acids and a missense mutation with a C0 score predicted to have low or null impact on protein function. However, whether these mutations were on different alleles could not be determined and one cannot exclude an undetected deep intronic mutation in this patient. Finally, case 53011A1 with early onset severe A-T presented residual P-KAP1 response. Altogether in this small series of A-T cases, it was difficult to correlate the clinical evolution from our biochemical and functional ATM characterization, and role of modifier genes cannot be excluded. Future evaluation of newly identified functions of $A T M, 8,9,27$ may give important clues for mutation/phenotype relationship.

In agreement with previous studies, ${ }^{19,28-32}$ we showed a decreased ATM protein in 14 of the $16 \mathrm{~A}-\mathrm{T}$ confirmed cell lines. Recent studies based on transfection of ATM constructs carrying various missense mutations showed that some missense mutations were associated with decreased ATM protein expression. ${ }^{17-19}$ The present study confirms that this phenomenon is also observed for endogenous ATM expression and is frequently associated with ATM missense mutations. Out of the two cases of our A-T series with normal ATM expression, one was bearing the c.6115G > A;p.Glu2039Lys mutation, which was modeled by Barone et $a l^{18}$ and shown to lead to a stable ATM protein with little kinase activity.

Nuclear localization of ATM is crucial to allow correct DNA damage signaling. ${ }^{22}$ A new finding of this study is the abnormal cytoplasmic localization of several mutated ATM products. The mechanisms underlying ATM localization have not yet been elucidated. Only one mutation in our series was located in the NLS of ATM (case 53011A1), and so impairment of the binding to importin could not be the general mechanism underlying our observation. Interestingly, our series include two changes of the same Asp2708 codon with different consequences: Asp2708Glu leading to a normal cytonuclear ratio, and Asp2708Asn - a nonpolar neutral versus acidic amino-acid change - to a cytoplasmic localization, suggesting that subtle conformation changes of the ATM protein may have marked consequences on its fate.

Out of the 14 A-T cell lines with underexpression, 13 presented a moderately to drastic abnormal cytoplasmic localization. Conversely, the two cases without ATM underexpression had normal protein localization. The link between abnormal cytoplasmic localization and decreased ATM expression is suggestive of exposure of cytoplasmic $A T M$ to proteases and subsequent ATM degradation. Previous studies have reported that $A T M$ can be a target for caspase 3 and caspase 7 under apoptotic conditions induced by irradiation or various agents, ${ }^{33-35}$ but inhibition of caspase activity failed to restore ATM protein level as well as the treatment by the export-inhibiting drug leptomycin B (Jacquemin et al, 2010 unpublished data). Alternatively, as recently reported, amino-acid changes can affect ATM conformation, post-translational modification or protein-protein interaction sites involved in protein localization and stability, ${ }^{18}$ but measured half life of mutated ATM proteins was not found decreased as compared with WT protein (Jacquemin et al, 2010 unpublished data). Furthermore case 32753A6 with major underexpression but normal localization and conservative amino-acid change indicates that each mutation could have peculiar consequences on ATM protein fate. However, understanding of the mechanisms underlying ATM underexpression and mislocalization could be critical to design new pharmacological strategies able to reverse these phenomena in A-T patients bearing missense mutations.

\section{CONFLICT OF INTEREST}

The authors declare no conflict of interest.

\section{ACKNOWLEDGEMENTS}

We thank Dr Debré, Dr Barbier, Pr Cosset, Pr Echenne, Dr Perelman, Dr Ponsot, Dr Pedespan, Dr Philip, Dr Leheup, Dr Jonyeaux, Dr Nguyen, Dr Delobel, Dr Guillot, Dr Soete, Dr Rivier and Dr Bordigoni for addressing patients, Genethon for access to cell lines, and the Institut Curie sequencing facility. VJ, SJ and GR are supported by grants from Association pour la Recherche sur le Cancer (ARC), from the Translational Department of Institut Curie, and the Ligue Nationale Contre le Cancer, respectively.

1 Boder E, Sedgwick RP: Ataxia-telangiectasia; a familial syndrome of progressive cerebellar ataxia, oculocutaneous telangiectasia and frequent pulmonary infection. Pediatrics 1958; 21: 526-554.

2 Chun HH, Gatti RA: Ataxia-telangiectasia, an evolving phenotype. DNA Repair (Amst) 2004; 3: 1187-1196.

3 Aurias A, Dutrillaux B, Buriot D, Lejeune J: High frequencies of inversions and translocations of chromosomes 7 and 14 in ataxia telangiectasia. Mutat Res 1980; 69: 369-374.

4 Savitsky K, Bar-Shira A, Gilad S et al: A single ataxia telangiectasia gene with a product similar to PI-3 kinase. Science 1995; 268: 1749-1753.

5 Lavin MF: Ataxia-telangiectasia: from a rare disorder to a paradigm for cell signalling and cancer. Nat Rev Mol Cell Biol 2008; 9: 759-769.

6 Watters D, Kedar P, Spring $\mathrm{K}$ et al: Localization of a portion of extranuclear ATM to peroxisomes. J Biol Chem 1999; 274: 34277-34282.

7 Li J, Han YR, Plummer MR, Herrup K: Cytoplasmic ATM in neurons modulates synaptic function. Curr Biol 2009; 19: 2091-2096.

8 Alexander A, Cai SL, Kim J et al: ATM signals to TSC2 in the cytoplasm to regulate mTORC1 in response to ROS. Proc Natl Acad Sci USA 2010; 107: 4153-4158.

9 Cam H, Easton JB, High A, Houghton PJ: mTORC1 signaling under hypoxic conditions is controlled by ATM-dependent phosphorylation of HIF-1alpha. Mol Cell 2010; 40: 509-520.

10 Wright J, Teraoka S, Onengut S et al: A high frequency of distinct ATM gene mutations in ataxia-telangiectasia. Am J Hum Genet 1996; 59: 839-846.

11 Concannon P, Gatti RA: Diversity of ATM gene mutations detected in patients with ataxia- telangiectasia. Hum Mutat 1997; 10: 100-107.

$12 \mathrm{Li} \mathrm{A}$, Swift M: Mutations at the ataxia-telangiectasia locus and clinical phenotypes of A-T patients. Am J Med Genet 2000; 92: 170-177.

13 Gilad S, Khosravi R, Shkedy D et al: Predominance of null mutations in ataxiatelangiectasia. Hum Mol Genet 1996; 5: 433-439. 
14 Micol R, Ben Slama L, Suarez F et al: Morbidity and mortality from ataxia-telangiectasia are associated with ATM genotype. J Allergy Clin Immunol 2011; 128: 382-389.

15 Stewart GS, Last JI, Stankovic T et al: Residual ataxia telangiectasia mutated protein function in cells from ataxia telangiectasia patients, with 5762 ins 137 and $7271 \mathrm{~T} \rightarrow \mathrm{G}$ mutations, showing a less severe phenotype. J Biol Chem 2001; 276: 30133-30141.

16 Verhagen MM, Abdo WF, Willemsen MA et al: Clinical spectrum of ataxia-telangiectasia in adulthood. Neurology 2009; 73: 430-437.

17 Mitui M, Nahas SA, Du LT et al: Functional and computational assessment of missense variants in the ataxia-telangiectasia mutated (ATM) gene: mutations with increased cancer risk. Hum Mutat 2009; 30: 12-21.

18 Barone G, Groom A, Reiman A, Srinivasan V, Byrd PJ, Taylor AM: Modeling ATM mutant proteins from missense changes confirms retained kinase activity. Hum Mutat 2009; 30: $1222-1230$.

19 Scott SP, Bendix R, Chen P, Clark R, Dork T, Lavin MF: Missense mutations but not allelic variants alter the function of ATM by dominant interference in patients with breast cancer. Proc Natl Acad Sci USA 2002; 99: 925-930.

20 Houdayer C, Dehainault C, Mattler C et al: Evaluation of in silico splice tools for decision-making in molecular diagnosis. Hum Mutat 2008; 29: 975-982.

21 Olive PL: Detection of DNA damage in individual cells by analysis of histone H2AX phosphorylation. Methods Cell Biol 2004; 75: 355-373.

22 Young DB, Jonnalagadda J, Gatei M, Jans DA, Meyn S, Khanna KK: Identification of domains of ataxia-telangiectasia mutated required for nuclear localization and chromatin association. J Biol Chem 2005; 280: 27587-27594.

23 Tavtigian SV, Oefner PJ, Babikyan D et al: Rare, evolutionarily unlikely missense substitutions in ATM confer increased risk of breast cancer. Am J Hum Genet 2009; 85: 427-446.

24 Porcedda $\mathrm{P}$, Turinetto $\mathrm{V}$, Brusco $\mathrm{A}$ et al: A rapid flow cytometry test based on histone $H 2 A X$ phosphorylation for the sensitive and specific diagnosis of ataxia telangiectasia. Cytometry A 2008; 73: 508-516.
25 Ziv Y, Bielopolski D, Galanty $Y$ et al: Chromatin relaxation in response to DNA double-strand breaks is modulated by a novel ATM- and KAP-1 dependent pathway. Nat Cell Biol 2006; 8: 870-876.

26 Isken O, Maquat LE: Quality control of eukaryotic mRNA: safeguarding cells from abnormal mRNA function. Genes Dev 2007; 21: 1833-1856.

27 Guo Z, Kozlov S, Lavin MF, Person MD, Paull TT: ATM activation by oxidative stress. Science 2010; 330: 517-521.

28 Stankovic T, Kidd AM, Sutcliffe A et al: ATM mutations and phenotypes in ataxiatelangiectasia families in the British Isles: expression of mutant ATM and the risk of leukemia, lymphoma, and breast cancer. Am J Hum Genet 1998; 62: 334-345.

29 Sandoval N, Platzer M, Rosenthal A et al: Characterization of ATM gene mutations in 66 ataxia telangiectasia families. Hum Mol Genet 1999; 8: 69-79.

30 Becker-Catania SG, Chen G. Hwang MJ et al: Ataxia-telangiectasia: phenotype/genotype studies of ATM protein expression, mutations, and radiosensitivity. Mol Genet Metab 2000; 70: 122-133.

31 Lakin ND, Weber P, Stankovic T, Rottinghaus ST, Taylor AM, Jackson SP: Analysis of the ATM protein in wild-type and ataxia telangiectasia cells. Oncogene 1996; 13: 2707-2716.

32 Angele S, Lauge A, Fernet $M$ et al: Phenotypic cellular characterization of an ataxia telangiectasia patient carrying a causal homozygous missense mutation. Hum Mutat 2003; 21: 169-170.

33 Smith GC, d'Adda di Fagagna F, Lakin ND, Jackson SP: Cleavage and inactivation of ATM during apoptosis. Mol Cell Biol 1999; 19: 6076-6084.

34 Tong $\mathrm{X}$, Liu B, Dong Y, Sun Z: Cleavage of ATM during radiation-induced apoptosis: caspase-3-like apoptotic protease as a candidate. Int J Radiat Biol 2000; 76: 1387-1395.

35 Hotti A, Jarvinen K, Siivola P, Holtta E: Caspases and mitochondria in c-Myc-induced apoptosis: identification of ATM as a new target of caspases. Oncogene 2000; 19: 2354-2362.

Supplementary Information accompanies the paper on European Journal of Human Genetics website (http://www.nature.com/ejhg) 